\title{
Biomarkers in Drug Free Subjects with Depression : Correlation with Tryptophan
}

\author{
Noreen Samad ${ }^{1}$, Farzana Yasmin ${ }^{2,3}$, and Natasha Manzoor ${ }^{1}$ \\ ${ }^{1}$ Department of Biochemistry, Bahauddin Zakariya University, Multan, Pakistan \\ ${ }^{2}$ Department of Biomedical Engineering, NED University of Engineering and Technology, Karachi, Pakistan \\ ${ }^{3}$ Department of Food Engineering, NED University of Engineering and Technology, Karachi, Pakistan
}

\begin{abstract}
Objective Depression is a prevalent condition that is costly to individuals and society. In view of a role of tryptophan (TRP), selenium $(\mathrm{Se})$, vitamin $\mathrm{D}(\mathrm{Vit} \mathrm{D})$, magnesium $(\mathrm{Mg})$ and serotonin in depression, the present study concerns to evaluate the circulating levels of TRP, Se, Vit D, Mg and serotonin in depression as well as the correlation between TRP and other serum analytes is also established.

Methods Healthy $(n=48)$ and depressed $(n=48)$ subjects were recruited and their blood samples were obtained after an overnight fast of $12 \mathrm{~h}$, serum was stored for the determination of levels of TRP, Se, Vit D, Mg, and serotonin.

Results Results show that levels of TRP, Se, Vit D, Mg, and serotonin were decreased in the depressed patient when compared to normal subjects. There is a direct correlation between TRP and Vit D, and TRP and Se while the inverse correlation between TRP and Mg, and TRP and serotonin in depressed subjects. The association among TRP and other biomarkers is non-significant.

Conclusion In conclusion, depression is associated with deficiency of TRP, Se, Vit D, Mg, and serotonin displays the characteristics of biomarkers. The correlation between TRP and other biomarkers/trace elements is also important in depression.
\end{abstract}

Psychiatry Investig 2019;16(12):948-953

Key Words Depression, Tryptophan, Selenium, Vitamin D, Serotonin, Magnesium, Trace elements, Biomarkers.

\section{INTRODUCTION}

Depression is a prevalent disease. ${ }^{1}$ While various treatment is effective with important limitation. The effects of medication produce in weeks; most of them have harmful effects and fail to produce preventive effects. ${ }^{2}$

Tryptophan (TRP) is an essential amino acid and is the predecessor of serotonin. Ogawa et al. ${ }^{3}$ studied that depressed patients had reduced plasma TRP levels. Previously it has been reported that depression is followed by elevated levels of glucocorticoids showed the hyperactivity of the hypothalamic-pituitary-adrenal (HPA)-axis. ${ }^{4}$

The bioactive neurotransmitter serotonin is derived from the essential amino acid TRP. Many studies have been prov-

Received: May 1, 2019 Revised: August 29, 2019

Accepted: September 20, 2019

$\triangle$ Correspondence: Noreen Samad, PhD

Department of Biochemistry, Bahauddin Zakariya University, Multan, Pakistan Tel: +0092-61-9210180, Fax: +0092-61-9210180

E-mail: noreen.samad@bzu.edu.pk

(c) This is an Open Access article distributed under the terms of the Creative Commons Attribution Non-Commercial License (https://creativecommons.org/licenses/by$\mathrm{nc} / 4.0$ ) which permits unrestricted non-commercial use, distribution, and reproduction in any medium, provided the original work is properly cited. en that the levels of serotonin were decreased in the brain ${ }^{5}$ and serum ${ }^{6}$ in depression. The hyperactivity of HPA-Axis due to depression not only leads the reduction of TRP levels in brain and serum ${ }^{7}$ as well involve in diminishing the levels of serotonin in the brain and serum.

Over the last decade deficiency of Vitamin D (Vit D) has established rising consideration. It is involved in the restoration of mood and other behaviors. Low level of Vit D observed in subjects with symptoms of depression. ${ }^{89}$ Recently, it is reported that depression is dependent on status of Vit $\mathrm{D}$, since its receptors are extensively scattered in areas of the human brain that are involved in major depressive disorder (MDD). ${ }^{10}$

Magnesium ( $\mathrm{Mg}$ ) is a plentiful mineral in the body and is crucial for good health. Mg is required for various biochemical reactions in the body. The post-injury administration of Mg efficiently enhanced upturn of cognitive decline. ${ }^{11}$ The presence of depressive symptoms in patients is also associated with $\mathrm{Mg}$ deficiency. ${ }^{12}$ and the high occurrence of $\mathrm{MDD}^{13,14}$ validate the assessment of changes in $\mathrm{Mg}$ concentration in the blood as a biomarker of depression.

Selenium (Se) is a vital trace element of primary significance to human wellbeing as it is the just one for which amalgama- 
tion into proteins is genetically encoded, as the important part of the amino acid selenocysteine. ${ }^{15}$ The selenocysteine has many physiological roles including being structural components of several antioxidant enzymes. ${ }^{16}$ Furthermore, reduced levels of Se have verified its role in mood disorders. ${ }^{17}$ Conner et al. ${ }^{18}$ recommended that reduce Se ingestion may be linked with a greater risk of depressive symptomatology even among healthy young adults.

Extensive studies have been reported that $\mathrm{TRP},{ }^{19} \mathrm{Mg},{ }^{20} \mathrm{Se},{ }^{21}$ Vit $\mathrm{D},{ }^{9}$ and serotonin ${ }^{7}$ play major role as biomarker in depression. TRP is an essential amino acid that has an important role in the human body and performs as a predecessor for a large variety of bio-chemicals, such as serotonin. Although much is known about the concentration and role of biomarkers in depression, the question, what is the correlation of TRP (a wellknown biomarker in depression) with other biomarkers (Vit D, Se, $\mathrm{Mg}$, serotonin) is still unknown, In an attempt to address this question the primary purpose of this study is to evaluate the serum TRP, Mg. Se, serotonin, Vit D in subjects with depression when compared with healthy volunteers. The secondary goal is to explore the potential association between TRP and other serum analytes in depressed subjects.

\section{METHODS}

The participants (depressed patients) were recruited from the outpatient of the psychiatric department of Nishtar medical college and Hospital Multan, Punjab, which belonged to Multan as well as other regions of southern Punjab, Pakistan. Total ninety six (96), Forty eight (48) healthy (un-depressed) and forty eight (48) depressed [83 (86.46\%) females and 13 (13.54\%) males] subjects of 35-65 years of age with normal body mass index (BMI) values $18.5-21.9 \mathrm{~kg} / \mathrm{m}^{2}$ were recruited in this study. Depressed patients were selected and included in this study after the diagnosis by the psychiatrist using DSM-IV criteria at the psychiatric department from March to June 2018. The severity of depression was evaluated using 17 item version Hamilton Depression Rating Sacale (HDRS-17) for depression. All subjects were up to date of the methods and goals of this study. Only participants who furnished written approval were selected. Ethical permission was obtained from the Departmental Ethics Committee (Ref No\# D-1884-Biochem, Dated: March 15, 2018).

Participants were disqualified from the study those were detected any other psychiatric illness; who had a history of taking the antidepressant, antipsychotics, corticosteroids, thyroxin, anticonvulsants, or estrogen; those who had aphasia; and who could not understand and answer the clinical questionnaires.

\section{Sample collection and biochemical estimations}

An overnight 12-h, fasting blood samples (at 7:00 am) were collected in 5-mL tubes within $24 \mathrm{hr}$ of completing the clinical questionnaires and were centrifuged (3000 rpm, $15 \mathrm{~min}$ utes, $4^{\circ} \mathrm{C}$ ). Serum was removed and then frozen at $70^{\circ} \mathrm{C}$ until the analysis of Vit D, Se, Mg, serotonin, and TRP. The techniques were used for research purpose high-performance liquid chromatography (HPLC) for finding out TRP levels and flame atomic absorption flame spectrophotometry (FAAS) for Se and $\mathrm{Mg}$ determination and enzyme-linked immunosorbent assay (ELISA) kits for serotonin and Vit D levels.

\section{Biochemical analysis}

\section{HPLC analysis}

Serum samples were extracted and the levels of serum TRP were determined by HPLC-EC. ${ }^{5}$ A $5 \mu \mathrm{m}$ Shim-Pack ODS separation column of $4.0 \mathrm{~mm}$ internal diameter and $150 \mathrm{~mm}$ length was used. Separation was achieved by mobile phase containing methanol (5\%), octyl sodium sulfate $(0.03 \%)$ and EDTA $(0.0035 \%)$ in $0.1 \mathrm{M}$ phosphate buffer of $\mathrm{pH} 2.9$ at an operating pressure 2,000-3,000 psi on Shimadzu HPLC pump. Electrochemical detection was achieved on Shimadzu L-ECD-6A detector at an operating potential of +0.8 to $1.0 \mathrm{~V}$.

\section{FAAS analysis}

Serum samples were diluted with $2 \%$ nitric acid and the levels of the serum $\mathrm{Mg}$ and Se were determined by FAAS. ${ }^{22}$

\section{ELISA}

Vit D and serotonin serum samples were determined by ELISA method using ELISA kits (Ann Arbor, Michigan, MI, USA). The kit method for determination of Vit D and serotonin was used as instructed.

\section{Statistical analysis}

Data on serum TRP, Vit D, Se, Mg and serotonin levels were analyzed by paired sample t-test. Pearson bivariate correlation and multiple regression were used to determined association among TRP and other biomarkers. All $\mathrm{p}$ values less than 0.05 were taken as significant.

\section{RESULTS}

Table 1 shows the demographic and clinical characteristics of participants including sex, age, BMI, age of onset of depression, sum score of disease severity and number of episode of depression of the 96 subjects participated in the present study.

Figure 1 shows the serum levels TRP, Se, Mg, Vit D and serotonin in depressed and normal subjects. Data analyzed 
by paired sample $\mathrm{t}$-test $(\mathrm{df}=47)$ showed that serum levels of TRP $(\mathrm{t}=13.37)$, Se $(\mathrm{t}=15.74), \mathrm{Mg}(\mathrm{t}=15.17)$, Vit $\mathrm{D}(\mathrm{t}=22.66)$ and serotonin $(\mathrm{t}=21.09)$ were significantly decreased in depressed subjects compared to normal volunteers. It is indicated that levels of TRP, Se, Mg, Vit D, and serotonin were decreased in depressed than healthy subjects.

Table 1. Demographic and clinical characteristics of the participants $(\mathrm{N}=96)$

\begin{tabular}{lcc}
\hline & Depressed (N=48) & Normal (N=48) \\
\hline $\mathrm{N}(\%)$ & $83(86.46)$ \\
Female & $13(13.54)$ \\
Male & $100(100)$ \\
Age (years) 35-65 & $19.1 \pm 1.3$ \\
Mean (SD) & N/A \\
BMI & $19.2 \pm 1.6$ & N/A \\
Total number of episodes & $4.36(3.24)$ & $3.92(2.81)$ \\
Age at onset (years) & $36(13.24)$ & \\
The severity of symptoms & & \\
The sum score of & $17.63(5.29)$ & \\
$\quad$ HDRS scores & & \\
\hline
\end{tabular}

SD: standard deviation, BMI: body mass index, HDRS: Hamilton Depression Rating Scale

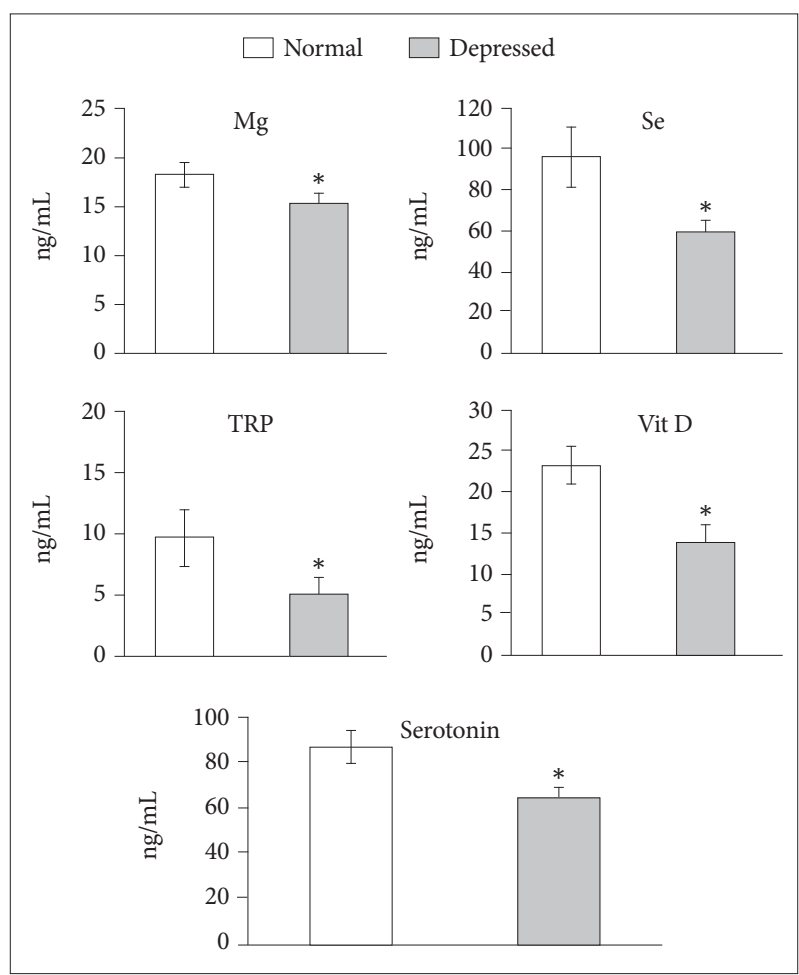

Figure 1. Shows the serum levels of TRP, Se, Vit D, Mg and serotonin in depressed and normal subjects. Values are mean \pm SD. Significant differences: ${ }^{*} p<0.05$ from respective normal following paired sample t-test. TRP: Tryptophan, Se: Selenium, Vit D: Vitamin D, Mg: Magnesium.
Figure 2 shows the correlation between TRP and Mg of depressed individuals. Data analyzed by Pearson bivariate correlation test showed that there is an inverse correlation ( $p>0.05 ; \mathrm{r}=-0.677)$ between two variables.

Figure 3 shows the correlation between TRP and serotonin of depressed subjects. Data analyzed by Pearson bivariate correlation test showed serotonin is inversely correlated ( $\mathrm{p}>0.05 ; \mathrm{r}=-0.109)$ with TRP.

Figure 4 shows the correlation between TRP and Se of depressed subjects. Data analyzed by Pearson bivariate correlation test showed an inverse correlation ( $\mathrm{p}>0.05 ; \mathrm{r}=0.250)$ between TRP and Se.

Figure 5 shows the correlation between TRP and Vit D of

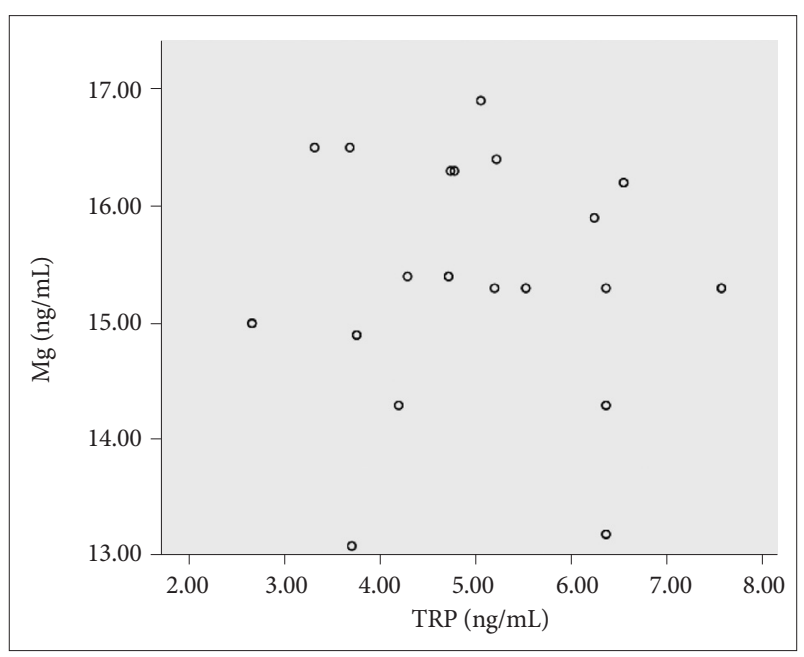

Figure 2. Correlation between serum TRP and Mg levels in patients with depression. Regression lines between the two variables are drawn to compare correlation. TRP: Tryptophan, Mg: Magnesium.

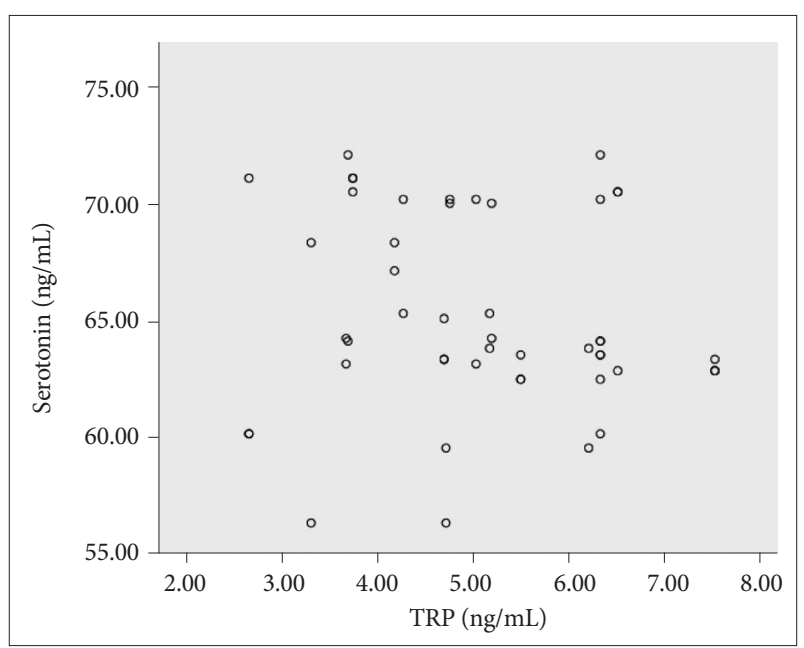

Figure 3. Correlation between serum TRP and serotonin levels in patients with depression. Regression lines between the two variables are drawn to compare correlation. TRP: Tryptophan. 


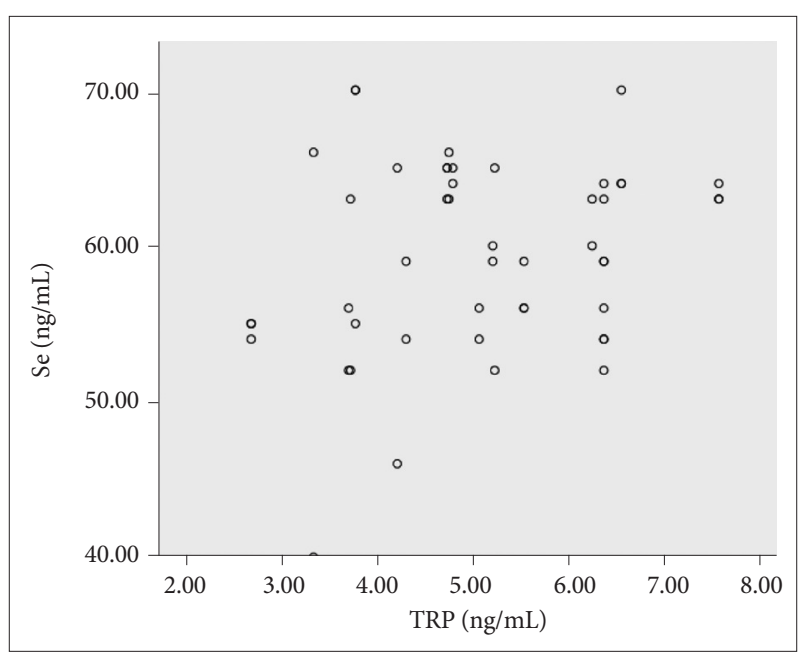

Figure 4. Correlation between serum TRP and Se levels in patients with depression. Regression lines between the two variables are drawn to compare correlation. TRP: Tryptophan, Se: Selenium.

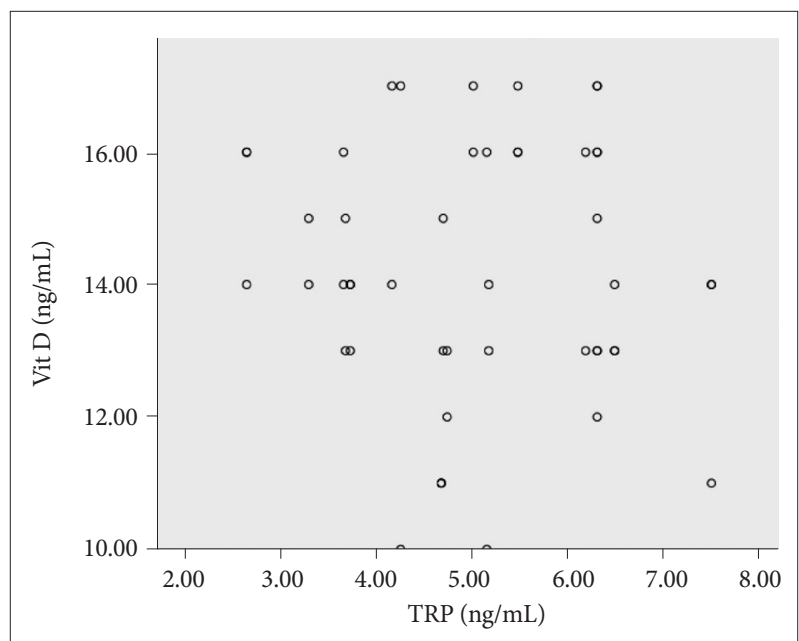

Figure 5. Correlation between serum TRP and Vit $D$ levels in patients with depression. Regression lines between the two variables are drawn to compare correlation. TRP: Tryptophan, Vit D: Vitamin D.

Table 2. Multiple regression analysis for the biomarkers data of depressed patients

\begin{tabular}{lccc}
\hline \multicolumn{1}{c}{ Variables } & $\begin{array}{c}\text { Standardized } \\
\text { coefficient }\end{array}$ & t value & p value \\
\hline Vitamin D & -0.041 & -0.271 & 0.781 \\
Selenium & 0.281 & 1.897 & 0.065 \\
Magnesium & -0.090 & -0.619 & 0.539 \\
Serotonin & -0.181 & -1.211 & 0.232 \\
\hline
\end{tabular}

$\mathrm{F}(4,43)=1.190, \mathrm{p}=0.329, \mathrm{R}^{2}=0.100$

depressed patients. Data analyzed by Pearson bivariate correlation test showed that there is a direct correlation $(\mathrm{p}<0.01$; $\mathrm{r}=0.497$ ) between TRP and Vit D.

Table 2 shows the evaluation whether TRP is associated with Vit D, Se, Mg and serotonin we performed an additional multiple regression analysis using data of depressed patients. The results showed that Vit D (0.701), Se (0.065), Mg (0.539) and serotonin (0.232) non-significantly associated with serum TRP levels.

\section{DISCUSSION}

Our data present that the number of male and female participant in the randomly selected Normal (non-depressed) and depressed groups, but there are more female than male in the depressed group (Table 1). In addition in this study we have selected normal BMI participant with greater number of women. The results support previous studies of greater occurrence of depression in female than male. ${ }^{23}$

Subsequent studies have shown that serum TRP levels were decreased significantly in depression. ${ }^{24}$ Observational studies and meta-analysis also showed that depression has an impact on serum analytes such as Vit $\mathrm{D},{ }^{7} \mathrm{Se},{ }^{18}$ serotonin ${ }^{24}$ and $\mathrm{Mg} .{ }^{14}$ It is also appeared from clinical data that the sum of HRDS score is greater in depressed than normal participants along with total number of episode (Table 1) that is also indicating that depression state influenced biochemical levels of various markers (Figure 1). The present study is aimed to find out the serum concentration of TRP, Vit D, Mg, Se and serotonin in depressed patients when compared to healthy volunteers. Apart from that we also correlated TRP which is a wellknown biomarker in depression with other serum Vit D, Mg, Se and serotonin in depressed patients.

In various studies low levels of serum Vit $\mathrm{D}, \mathrm{Se}^{18}$ serotonin ${ }^{6}$ and $\mathrm{Mg}^{14}$ have been reported in depression, which is also consistent with our study (Figure 1). Most important an inverse correlation was found between TRP and Mg (Figure 2 ) and TRP and serotonin (Figure 2), whereas a direct correlation was found between TRP and Se (Figure 3) and TRP and Vit $\mathrm{D}$ (Figure 5). In addition association among TRP and Vit D, Se, Mg and TRP was non substantial when performed with multiple regression analysis (Table 2), it is indicated, that reduced levels of Se, serotonin, $\mathrm{Mg}$ and Vit D have strong correlation with reduced levels of TRP in depression (Figs. 2-5, Table 2). The present finding suggests that not only TRP, other serum analytes such as Se, serotonin, $\mathrm{Mg}$ and Vit $\mathrm{D}$ are potential biomarkers in depression.

Lower levels of serum Vit D have been associated with various diseases. ${ }^{25}$ In the present study, decreased levels of Vit D (Figure 1) also suggested its role in depression. The mechanism linking in Vit D and TRP in depressed subjects is not well known, but there are numerous probable biochemical mechanisms involved by which association of Vit $\mathrm{D}$ and TRP could change the manifestation of depression. Studies have 
shown that TRP levels become decreased in depression because of the activation of HPA-axis. ${ }^{26}$ TRP is the precursor of an important neurotransmitter serotonin. Previously it was documented that Vit $\mathrm{D}$ up-regulate serotonin synthesis by activating the transcription of the serotonin synthesizing enzymes TRP hydroxylase in the brain. So it is validated by our study that TRP and Vit D (Figure 5, Table 2) have a potential association for the causal of depression.

Se is an essential trace element for humans and animals, needed for a broad variety of physiological functions. Se is also crucial for the living organism due to its antioxidant potential against oxidative damage. Much of this beneficial impact is attributed to seleno-enzymes, like glutathione peroxidases and thioredoxin reductases, in which Se has its site, Se may have some other special functions as well. ${ }^{27}$ Studies have revealed that state of depression may occurred due to free radicals/pro-oxidant production and antioxidant such as $\mathrm{Se}$ has role against free radicals induced oxidative stress. ${ }^{28}$ Protective effects of Se may also be attributed to its ability to modulate an anti-inflammatory effect..$^{28}$ Several hypotheses link selenium and other micronutrients with the pathophysiology of depression. ${ }^{21}$ Depression was significantly associated with low selenium blood level $\mathrm{s}^{29}$ and low levels of dietary Se were also associated with an increased risk for the major depressive disorder. ${ }^{21}$ The presented work is also in agreement with previous work ${ }^{21,29}$ that reduced levels of Se are linked with depression. The association between TRP and Se is positive point out that hyperactivity of HPA-axis not only reduced the levels of TRP in the brain and plasma but also cause the oxidative stress, which could be influenced on the antioxidant mechanism. Extensive studies have documented that $\mathrm{Se}$, is the part of antioxidant defense system, ${ }^{27}$ so the oxidants produced due to depression they also effect on the selenoprotein and also reduced its peripheral levels. So it is also validated that Se and TRP (Figure 4, Table 1) has a direct link in depression and Se has also stated a biomarker of depression.

Mg involves in many physiological processes. ${ }^{30}$ Neuroprotective effects of $\mathrm{Mg}$ are well-known ${ }^{31}$ and accord to psychiatric disorders particularly in the pathophysiology of depression..$^{32}$ Lower levels of serum $\mathrm{Mg}$ depression were published in subsequent studies ${ }^{31,32}$ which is consistent with our work (Figure 1). There is an association between the catecholaminergic system and the metabolism of Mg. Researchers, indicate that, $\mathrm{Mg}$ deficiency decreases the metabolism of catecholamines, causing an increase in psychomotor activity, while hypermagnesemia can cause the opposite effect. ${ }^{33}$ It is well known that Mg has a vital role in the metabolism of TRP and role of both Mg and TRP in depression is well documented. The present results revealed that the correlation between TRP and $\mathrm{Mg}$ is directly proportional and the reduced levels of both have potential effect in the induction of depression.

Serotonin is resulting from TRP. ${ }^{34}$ Depression is a neurobiological disorder linked with neurochemical, neuroendocrine and neuroimmunological dis-functioning. Low CSF levels of the serum 5-hydroxyl indoleacetic acid (5 HIAA) is allied with higher lifetime aggressivity, impulsivity and greater suicidal intent in patients with MDD. ${ }^{35}$ Previously it has also been reported that serum serotonin levels become reduced due to depression, ${ }^{35}$ which is in agreement with our study (Figure 1). Extensive studies have reported that TRP is the precursor of serotonin $34,{ }^{35}$ and the hyperactivity of HPA-axis in depression decreases the levels of TRP which in turn decreases the synthesis of serotonin in the brain as well as in the serum. ${ }^{7}$ It is suggested from the present study that TRP and serotonin have strong relationship and serotonin is dependent on the availability of TRP, and both of the serum analytes are strong biomarkers for the diagnosis of depression.

In conclusion, the present study shows that depression reduces the circulating levels of Vit D, TRP, Se, Mg and serotonin. Despite all the fact we also confirm that Vit D, TRP, Se, $\mathrm{Mg}$ and serotonin levels display characteristics of biomarkers. The association between TRP and others biomarkers is much strong in depression. The findings may help to improve understanding of biomarkers and therapeutics in depression.

\section{Conflicts of Interest}

The authors have no potential conflicts of interest to disclose.

\section{Author Contributions}

Conceptualization: Noreen Samad. Data curation: Farzana Yasmin, Natasha Manzoor. Formal analysis: Noreen Samad, Natasha Manzoor. Investigation: Noreen Samad, Natasha Manzoor. Methodology: Noreen Samad, Farzana Yasmin. Supervision: Noreen Samad. Writing-Original draft: Noreen Samad. Writing_review \& editing: Noreen Samad.

\section{ORCID iDs}

Noreen Samad https://orcid.org/0000-0003-4762-1667

\section{REFERENCES}

1. Centers for Disease Control and Prevention. Current depression among adults-United States, 2006 and 2008. MMWR Morb Mortal Wkly Rep 2010;59:1229-1235.

2. Zarate C, Duman RS, Liu G, Sartori S, Quiroz J, Murck H. New paradigms for treatment-resistant depression. Ann N Y Acad Sci 2013; 1292:21-31.

3. Ogawa S, Fujii T, Koga N, Hori H, Teraishi T, Hattori K, et al. Plasma Ltryptophan concentration in major depressive disorder: new data and meta-analysis. J Clin Psychiatry 2014;75:906-915.

4. Carroll BJ. Dexamethasone suppression test in depression. Lancet 1980;2:1249.

5. Samad N, Batool F, Haleem DJ. Neurochemical and behavioral effects of 8-OH-DPAT following exposure to restraint stress in rats. Pharmacol Rep 2007;59:173-180.

6. Saldanha BD, Kumar MN, Ryali VSSR, Srivastava K, Pawar AA. Serum serotonin abnormality in depression. Med J Armed Forces India 2009; 65:108-112. 
7. Maes M, Leonard BE, Myint AM, Kubera M, Verkerk R. The new '5HT' hypothesis of depression: cell-mediated immune activation induces indoleamine 2,3-dioxygenase, which leads to lower plasma tryptophan and an increased synthesis of detrimental tryptophan catabolites (TRYCATs), both of which contribute to the onset of depression. Prog Neuropsychopharmacol Biol Psychiatry 2011;35:702-721.

8. Lee MD, Tajar A, O’Neill WT, O’Connor BD, Bartfai G, Boonen S, et al. Lower vitamin D levels are associated with depression among community-dwelling European men. J Psychopharmacol 2011;25:1320-1328.

9. Lapid MI, Cha SS, Takahashi PY. Vitamin D and depression in geriatric primary care patients. Clin Interv Aging 2013;8:509-514.

10. Berk M, Sanders KM, Pasco JA, Jacka FN, Williams LJ, Hayles AL, et al. Vitamin D deficiency may play a role in depression. Med Hypotheses 2007;69:1316-1319.

11. Hoane MR. Assessment of cognitive function following magnesium therapy in the traumatically injured brain. Magnes Res 2007;20:229-236.

12. Eby GA, Eby KL. Rapid recovery from major depression using magnesium treatment. Med Hypotheses 2006;67:362-370.

13. Sowa-Kucma M, Szewczyk B, Sadlik K, Piekoszewski W, Trela F, Opoka $\mathrm{W}$, et al. Zinc, magnesium and NMDA receptor alterations in the hippocampus of suicide victims. J Affect Disord 2013;151:924-931.

14. Markkula N, Suvisaari J, Saarni SI, Pirkola S, Pena S, Saarni S, et al. Prevalence and correlates of major depressive disorder and dysthymia in an eleven-year follow-up-results from the Finnish Health 2011 Survey. J Affect Disord 2011;173:73-80.

15. Roman M, Jitaru P, Barbante C. Selenium biochemistry and its role for human health. Metallomics 2014;6:25-54.

16. Młyniec K, Gaweł M, Doboszewska U, Starowicz G, Pytka K, Davies $\mathrm{CL}$, et al. Essential elements in depression and anxiety. Part II. Pharmacol Rep 2015;67:187-194.

17. Sher L. Depression and suicidal behavior in alcohol abusing adolescents: possible role of selenium deficiency. Minerva Pediatr 2008;60:201-209.

18. Conner TS, Richardson AC, Miller JC. Optimal serum selenium concentrations are associated with lower depressive symptoms and negative mood among young adults. J Nutr 2015;145:59-65.

19. Ogawa S, Fujii T, Koga N, Hori H, Teraishi T, Hattori K, et al. Plasma L-tryptophan concentration in major depressive disorder: new data and meta-analysis. J Clin Psychiatry 2014;75:906-915.

20. Saveanu RV, Nemeroff CB. Etiology of depression: genetic and environmental factors. Psychiatr Clin North Am 2012;35:51-71.

21. Pasco JA, Jacka FN, Williams LJ, Evans-Cleverdon M, Brennan SL, Kotowicz MA, et al. Dietary selenium and major depression: a nested case-control study. Complement Ther Med 2012;20:119-123.

22. Naureen F, Masroor K, Khatoon F, Ayub S, Ahmed MI, Hasnat A, et al.
Zinc and tryptophan levels in anorexia nervosa: a co-relational study. Int J Biomed Eng Sci 2014;1:27-33.

23. Haleem DJ, Sheikh S, Fawad A, Haleem MA. Fasting leptin and glucose in normal weight, over weight and obese men and women diabetes patients with and without clinical depression. Metab Brain Dis 2017;32:757-764.

24. Karu N, McKercher C, Nichols DS, Davies N, Shellie RA, Hilder EF, et al. Tryptophan metabolism, its relation to inflammation and stress markers and association with psychological and cognitive functioning: Tasmanian Chronic Kidney Disease pilot study. BMC Nephrol 2016;17:171.

25. Holick MF. Vitamin D deficiency. N Engl J Med 2007;357:266-281.

26. Lin YT, Liu TY, Yang CY, Yu YL, Chen TC, Day YJ, et al. Chronic activation of NPFFR2 stimulates the stress related depressive behaviors through HPA axis modulation. Psychoneuroendocrinology 2016;7:73-85.

27. Solovyev ND. Importance of selenium and selenoprotein for brain function: from antioxidant protection to neuronal signaling. J Inorg Biochem 2015;153:1-12.

28. Hagmeyer S, Mangus K, Boeckers TM, Grabrucker AM. Effects of trace metal profiles characteristic for autism on synapses in cultured neurons. Neural Plasticity 2015;2015:985083.

29. Ibarra O, Gili M, Roca M, Vives M, Serrano MJ, Pareja A, et al. The mediterranean diet and micronutrient levels in depressive patients. Nutr Hosp 2014:31:1171-1175.

30. Cheng SM, Yang DU, Lee CP, Hung-Chuan Pan HC, Lin MT, Chen $\mathrm{SH}$, et al. Effects of magnesium sulfate on dynamic changes of brain glucose and its metabolites during a short-term forced swimming in gerbils. Eur J Appl Physiol 2007;99:695-699.

31. Xu ZP, Li L, Bao J, Wang ZH, Zeng J, Liu EJ, et al. Magnesium protects cognitive functions and synaptic plasticity in streptozotocin-induced sporadic Alzheimer's model. PLoS One 2014;9:e108645.

32. Singewald N, Sinner C, Hetzenauer A, Sartori SB, Murck H. Magnesium-deficient diet alters depression- and anxiety-related behavior in mice-influence of desipramine and Hypericum perforatum extract. Neuropharmacology 2004;47:1189-1197.

33. Widmer J, Henrotte JG, Raffin Y, Bovier P, Hilleret H, Gaillard JM. Relationship between erythrocyte magnesium, plasma electrolytes and cortisol, and intensity of symptoms in major depressed patients. J Affect Disord 1995;34:201-209.

34. Fernstrom JD. Role of precursor availability in control of monoamine biosynthesis in brain. Physiol Rev 1983;63:484-546.

35. Placidi GP, Oquendo MA, Malone KM, Huang YY, Ellis SP, Mann JJ. Aggressivity, suicide attempts, a relationship to cerebrospinal fluid monoamine metabolite levels. Biol Psychiatry 2001;50:783-791. 